\title{
Human Face Detection: Manual vs. Kohonen Self Organizing Map
}

\author{
Payal Bose $^{\mathrm{a} *}$, Prof. Samir K Bandyopadhyay ${ }^{\mathrm{b}}$ \\ ${ }^{a}$ Research Scholar at Lincoln University College, Lembah Sireh, 15050 Kota Bharu, Kelantan, Malaysia \\ ${ }^{b}$ Distinguish Professor at Lincoln University College, Lembah Sireh, 15050 Kota Bharu, Kelantan, Malaysia \\ ${ }^{a}$ Email: bosepayal91@gmail.com \\ ${ }^{b}$ Email:1954samir@gmail.com
}

\begin{abstract}
In today's world it is very much important to maintain the security of information and its risks. The biometricbased techniques are very much useful in these problems. Among the several kinds of biometric-based technique, face detection is much complex and much more important. Due to the age and several other problems, a human face structure changes over time, again a human has lots of expressions. Sometimes due to the lighting condition or the variation of the angle of an input device, the pattern of a human face structure also changed. As a result, the face cannot be detected properly. In this paper, a method is proposed that can detect the human faces both automatically and manually very efficiently. In manual mode, a user can select the input faces referred by the system according to their choice. In automated mode, the system detected all possible face areas using the Kohonen Self-Organizing Feature Map technique. This method reduced the complex color image into a vector quantized image with desired colors. Then a color segmentation technique is used to detect the possible face skin areas from the vector quantized image. Then the Histogram Oriented Gradient technique used to detect the feature from the faces and K-Nearest Neighbor Classifier is used to compare both face images detected by the two modes. The automated method prosed better accuracy than the manual method.
\end{abstract}

Keywords: Face Detection; Kohonen Self-Organizing Feature Map (K-SOM); Skin Color Segmentation; KNearest Neighbor (KNN) Classifier.

* Corresponding author. 


\section{Introduction}

In recent years the biometric-based recognition techniques are more promising than individual persons' authentication and granting them to access the domain based on password input. Biometric-based recognition techniques include facial identification, fingerprint identification, iris detection, palm detection, etc. Among these several techniques, the face detection technique is most important. The face detection technique has more advantages than other biometric-based recognition techniques. Because this process can be done passively without notifying the participant since the face image can be captured by a camera from a distance. This technique is especially suitable for security and surveillance purpose. The face detection system can be done by analyzing several algorithms. Human skin color segmentation technique is one of the most prominent techniques to detect a face accurately because a human face contains skin. In this paper, a method is developed that segmented the human skin and skin-like regions efficiently by both manually and automatically. Then a classifier is used to compare the accuracy of both manual, and automatic mode.

\section{Existing Work}

In image processing color segmentation is very much important. For a face detection system, skin color segmentation is much more efficient than any other face detection method. There are several kinds of face detection systems available based on color segmentation. The authors proposed a method that automatically classifies the colors on printed fabric by using the self-organizing method. This method partition the different color regions accurately. Firstly, this method identified the primary colors of the input image and then flagged by using the SOM's density map and U-matrix. Each color region is located by dividing the U-matrix with an adaptive threshold. This adaptive threshold value changes its value based on the network to get a perfect value. Finally, the high color regions are were segmented. Unlike other methods, this process doesn't need predefined cluster value, it auto-detect them from U-matrix [1]. Again the authors proposed a face recognition system based on Principal Component Analysis (PCA) for feature selection and Kohonen Self Organizing Map (K-SOM) for classification. The proposed system has the accuracy of the face detection system is $97 \%$ and recognition accuracy is $86.84 \%$ [2]. The authors proposed a multistage K-Nearest Neighbor classifier that collaborated with the coding based Bag-of-Features to solve the SSPP problem. Here first they use K-means to collect the local features, then use KNN to project the feature into a semantic space. Finally, an SVM classifier is used to concatenate the features for classifying the images. Three public databased was used to verifying the method. This method has great robustness towards expression, illumination, the occlusion, and, the time variation [3]. Again the authors proposed a method that solves the attendance problem in college and schools. The used Linear Discriminant Analysis (LDA) with the K-Nearest Neighbor (KNN) algorithm to solve the face recognition problem [4].

\section{Proposed Methodology for Human face Detection}

In this paper, the face detection process is divided into two parts, 1) Manual mode, 2) Automated mode. In manual mode, the user can able to select the human faces present in the input image. And in automated mode, the Kohonen self-organizing feature map is used to detect the human faces present in the input image. The steps 
of the proposed method for face detection are shown in figure-1.

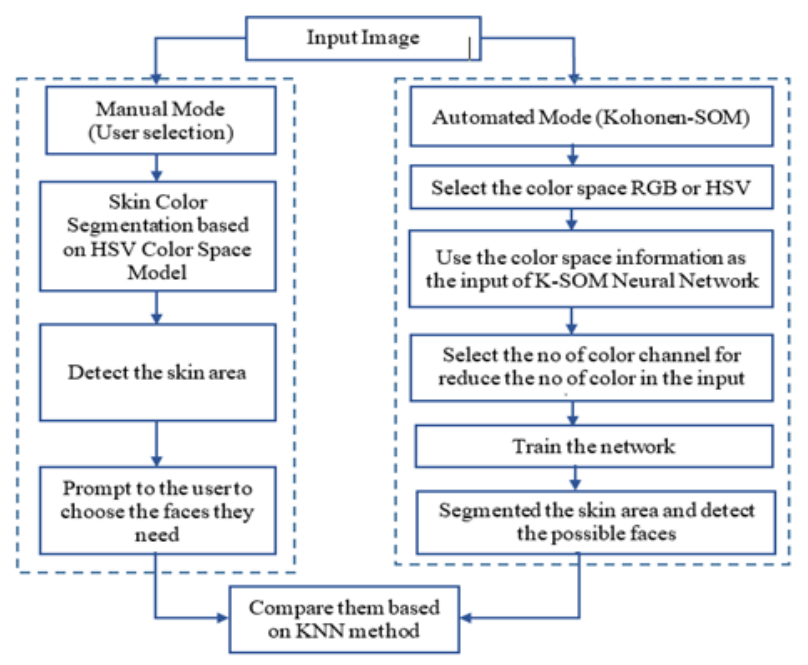

Figure 1: Face Detection Procedure (Manual and Automated)

\subsection{Manual Face Detection Procedure}

In this process first, the system finds out all possible faces. Second, the user selects the particular face or faces according to their choice. To find out the possible faces, here well-known HSV color space segmentation technique [5] is used. HSV, Hue, Saturation, and Value, color space model can efficiently separate the chrominance and luminance color variation and the colors remain unchanged in different lighting conditions. For this reason HSV color space model popular for the skin detection method. For skin color detection first, convert the input color image into HSV color space image. Second, segment all the color channels with a certain range of threshold values. The range for thresholding for each channel is given below:

$$
\begin{gathered}
H_{\text {thresh }}=0.40<h<0.50 \\
S_{\text {thresh }}=0.65<s<0.74 \\
V_{\text {thresh }}=0.56<v<0.65
\end{gathered}
$$

Next, combine all the segmented channels to detect the skin area. The result is shown in figure-2.

$$
H S V_{\text {seg }}=\text { combine }\left(H_{\text {thresh }}, S_{\text {thresh }}, V_{\text {thresh }}\right)
$$

The next, step is to prompt the user which skin area they want to accept as a face. After selecting the face area, the selected area is bounded by a bounding box with the color red to separate from other bounding boxes. If the user does not accept any region as a face then the system detects the next region and prompts again. This process will continue until it covers all the skin like areas. Finally, all the selected faces by the user, stored in a temporary folder for the next process. This process is shown in figure-3. 


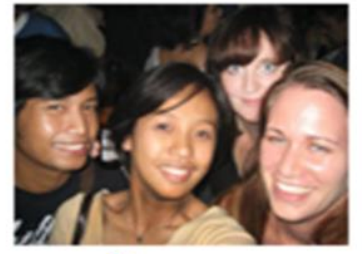

a

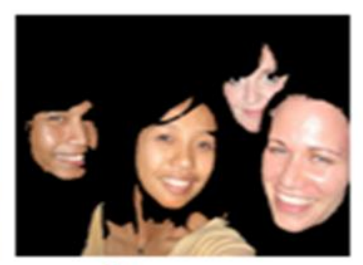

b

Figure 2: (a) Original image, (b) Detected possible skin area

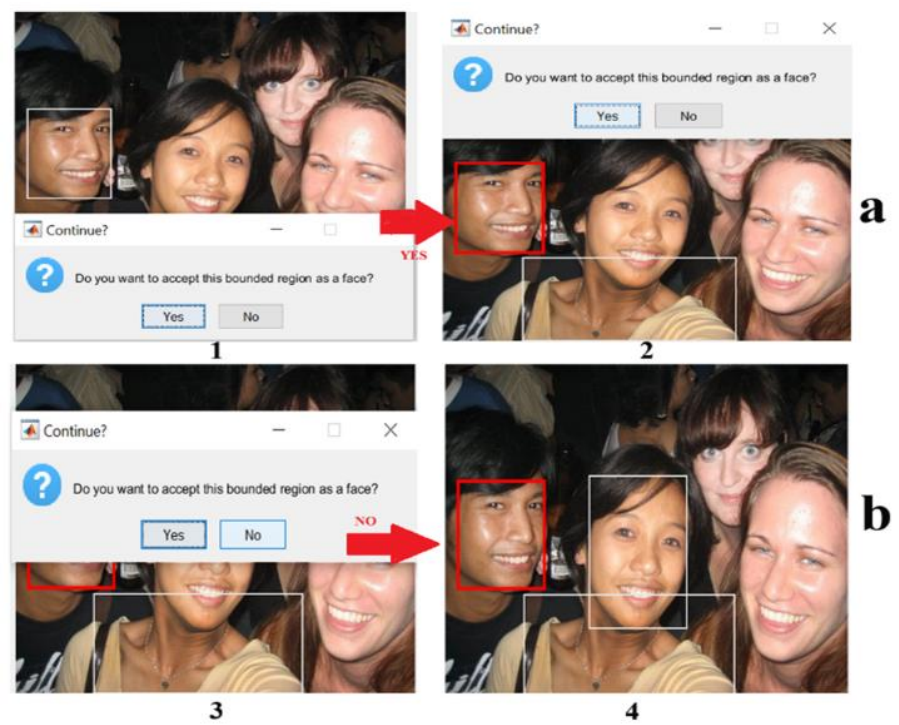

Figure 3: (a.1) when the user chooses the detected skin area as a tace (a.2) Atter acceptıng the skin area like face, the area marked with a red color box and detect the next skin area (b.3) when the user doesn't want to choose the detected skin area as a face (b.4) Next skin area detected

All the skin areas accepted by the user as a face is shown in figure-4.
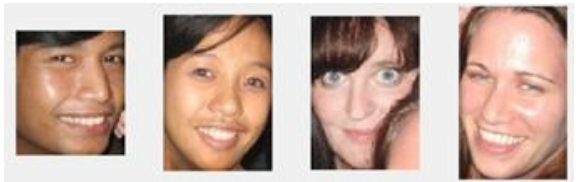

Figure 4: Faces detected by the user

\subsection{Automated Face Detection Procedure}

In this paper, for automated face detection, Kohonen self-organizing feature map is used (K-SOFM) [1-2,6]. First, this process selects the color space model, RGB, or HSV. Then use that color space information to detect the skin and skin-like regions using K-SOFM. This process helps to reduce the complexity of color regions to simple color regions. So that the detection of the proper skin and skin-like regions is very efficient.

\subsubsection{Kohonen Self-Organizing Feature Map}


The self-organizing map (SOM) [7] was introduced by Teuvo Kohonen. This is an unsupervised learning process. It learns the patterns from a set of unknown data without knowing any class information. It is a fully connected single-layer neural network. SOFM created two-dimensional output which is known as a map. It is a very high stable neural network. Unlike other neural networks, SOFM selected the suitably optimized output nodes with closely related to the training sample data for the class of input vector. This is the reason SOFM doesn't need any target output node. The Kohonen learning algorithm[8,9] creates a vector quantizer by repeat updating the sample of input classes. Among the input samples, which are called neurons of the SOFM neural network $(\mathrm{NN})$, there is a competition to be activated first. Finally, only one neuron win the competition. The steps followed by the K-SOFM for this experiment is given below:

Step-1: Initialize the learning rate $l_{r}(t)=0.001$, weight $W_{i}(t)=$ random and network size or neighbors $N_{i}(t)=$ based on user choice, Example: if the user wants to reduce the no of colors in the image is $n$ Color then $N_{i}(t)$ calculated as

$$
N_{i}(t)=[\text { floor }(\sqrt{ }(n \text { Color })) \times \operatorname{ceil}(\sqrt{ }(n \text { Color }))]
$$

Step-2: Collect the input patterns $I_{p}$ from color channels of the color space model (RGB or HSV)

Step-3: Start training the network and determine the winning class which is closest to the $I_{p}$.

$$
d=\min \left(/ / W_{i}(t)-I_{p} / /\right)
$$

Step-4: Update $W_{i}(t), l_{r}(t), N_{i}(t)$

$$
\begin{aligned}
& W_{i}(t)=W_{i}(t+1)+l_{r}(t), \\
& l_{r}(t)=l_{r}(t+1), \\
& N_{i}(t)=N_{i}(t+1)
\end{aligned}
$$

Step-5: Repeat the process until reach the maximum epoch. The result after applying the K-SOFM to the input image is shown in figure-5.

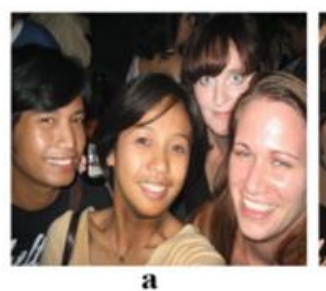

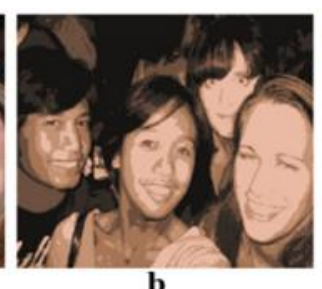

$\mathbf{b}$

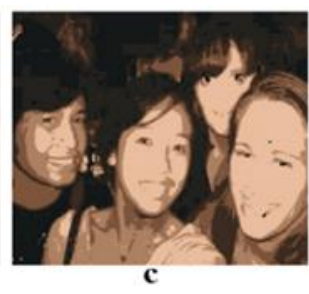

Figure 5: a) Original Input Image, b) Reduce the no of the color channel (here 6) for RGB color space image, c) Reduce the no of the color channel (here 6) for HSV color space image 


\subsubsection{Skin Segmentation}

After reducing the number of colors, the next step is to segment the true skin regions[5,10] to detect the face. In this experiment two, color space models RGB, and HSV are used. For color space, HSV uses equations 1, 2, 3, and 4 to detect the skin area. For color space, RGB the range of thresholding for each channel is given below:

$R_{\text {thresh }}=0.60<r<0.68$

$G_{\text {thresh }}=0.24<g<0.29$

$B_{\text {thresh }}=0.12<b<0.18$

Next, combine all the segmented channels to detect the skin area. The result is shown in figure- 2 .

$R G B_{\text {seg }}=$ combine $\left(R_{\text {thresh }}, G_{\text {thresh }}, B_{\text {thresh }}\right)$

The experimental results are shown in figure-6.

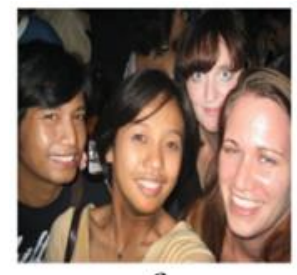

a

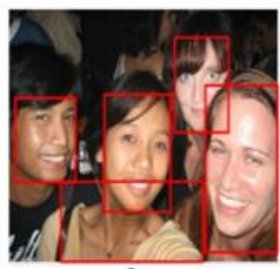

b

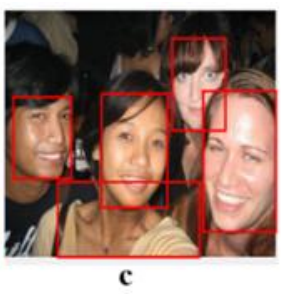

Figure 6: a) Original Image, b) Detected skin regions after RGB color segmentation, c) Detected skin regions after HSV color segmentation

3.2.3. Comparison between Manual and Automated face detection Using Histogram Gradient Oriented (HOG) feature extraction and K Nearest Neighbor (KNN) Algorithm

Histogram Gradient Oriented Method [11,12] is one of the most commonly used features selection method. It broke the entire image into small regions and then calculate the gradient and orientation of each region and finally calculated the histogram of them. The three main steps of the process are, 1) Prepossessing the data, 2) Calculate gradients, 2) then calculate the magnitude and orientation.

Magnitude $=\sqrt{ }\left(g_{x}^{2}+g_{y}^{2}\right)$

Orientation $(\varnothing)=\arctan \left(g_{x} / g_{y}\right)$

Where, $\mathrm{g}_{\mathrm{x}}$, and, $\mathrm{g}_{\mathrm{y}}$ are the gradient values along $\mathrm{x}$ and $\mathrm{y}$ direction.

K-nearest neighbor $(\mathrm{KNN})[3-4,13,14]$ is one of the most basic and essential classification algorithms in machine learning. It belongs to the supervised learning method. This classifier is mostly used in pattern 
recognition. To classify the unknown sample, this classifier calculates the distance between the unknown sample and all datasets. The smallest value distance between the unknown sample and the training set is accepted. Then the unknown sample as classified by its nearest neighbor. The performance of KNN depends on the value of K, the no of nearest neighbor, to classify an unknown sample. In this experiment, the image size $[200 \times 150]$ was taken for the HOG feature calculation. Manually selected faces are used as training sample and automated selected faces are test data. The experimental results are shown in figure-7.

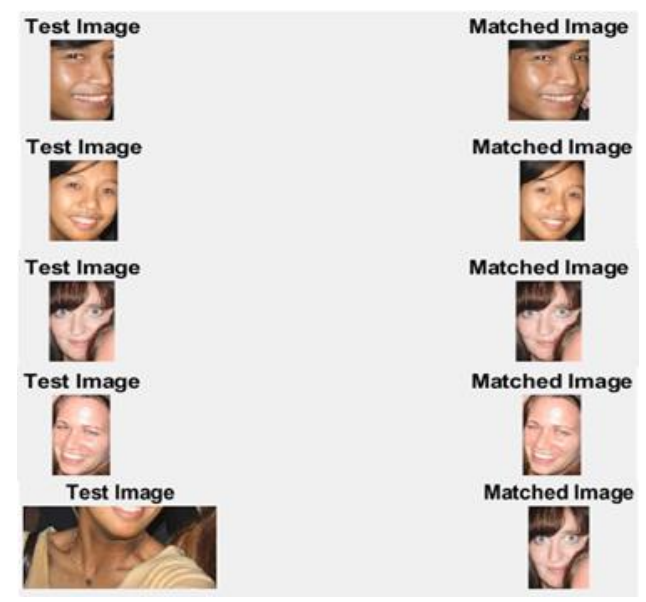

Figure 7: Experimental Result of Comparison between Manual (Training) and Automated (Test) face detection based on KNN Classifier.

\section{Performance Analysis and Discussions}

This experiment has two major part one, detect a face in manual mode, and two, detect a face in automated mode. Here for experimental purposes, the maximum group of two to four-person images was accepted. In automated mode first, the system uses the K-SOM to reduce the complex color input image into a vector quantized image. Then use a skin color segmentation process to detect the possible faces in the input image. Here also Fuzzy C-Means [15] and K-Means [16] clustering techniques were used to compare the cluster analysis among the three techniques. The experimental result is shown in table 1.

Table 1: The Experimental Result of Different Clustering Method

\begin{tabular}{l|l|l|l}
\hline \multirow{2}{*}{ Method } & \multicolumn{2}{|l|}{ Accuracy of Cluster Analysis (\%) } & \multirow{2}{*}{ Accuracy of } \\
\cline { 2 - 3 } & $\begin{array}{l}\text { no of iteration } \\
\text { no of iteration } \\
\text { face } \\
\text { detection } \\
\end{array}$ & 900 & $(\%)$ \\
\hline K-SOM & 89.22 & 90.15 & 90.33 \\
K-Means & 92.34 & 93.01 & 94.28 \\
Fuzzy C Means & 87.28 & 88.56 & 89.78 \\
\hline
\end{tabular}


In manual mode first, the system performs skin color segmentation then suggests possible faces to the user. Then the user selects those faces that are required. After that, the system compares both manual and automated detected faces to check whether they matched correctly. Here the system uses the KNN classifier to compare the images. The experimental results are shown in table-2.

Table 2: Experimental result for system suggested and user accepted no of faces in manual mode

\begin{tabular}{llllr}
\hline Accuracy of Manual & Accuracy of & Manual & Accuracy & of \\
detected Faces $(\%)$ & detected Faces $(\%)$ & Comparison & using \\
& & KNN & \\
\hline 93.33 & 98.33 & 94.28 & \\
\hline
\end{tabular}

In this experiment, high-resolution images were used. The limitations of this experiment are, in manual mode users only selected among those face images that are suggested by the system. The input image needs to contain 2or 3 or 4 persons only.

\section{Conclusion}

In this paper, a face detection system is proposed that detects the human faces from an input image both manually and automatically. In manual mode face detection, the users can select the human faces from the input image that are suggested by the system. In automated detection first, an unsupervised learning technique Kohonen Self-Organizing Feature Map is used to convert the complex color input image into a vector quantized image. Then detect the possible faces in that image by using a skin color segmentation technique. After that, compare both manual and automated detected faces by using the HOG features detection technique and KNearest Neighbor classifier.

\section{References}

[1]. H. Mo, B. Xu, W. Ouyang, and J. Wang, "Color segmentation of multi-colored fabrics using selforganizing-map based clustering algorithm," Text. Res. J., vol. 87, no. 3, pp. 369-380, 2017, doi: $10.1177 / 0040517516631307$.

[2]. S. S. Sofi and R. A. Khan, "Face recognition using neural network technique som (Self organizing maps),” Int. J. Sci. Technol. Res., vol. 8, no. 12, pp. 3423-3427, 2019.

[3]. F. Liu, S. Yang, Y. Ding, and F. Xu, "Single sample face recognition via BoF using multistage KNN collaborative coding," Multimed. Tools Appl., vol. 78, no. 10, pp. 13297-13311, 2019, doi: 10.1007/s11042-018-7002-5.

[4]. S. S. Kumar, P. K. Sahoo, and K. Eswaran, “Class Room Attendance System Using KNN,” no. April, 2019.

[5]. B. S. Sathish, "Segmentation in RGB and HSV Color Space,” pp. 2-7, 2015.

[6]. S. Ghorpade, J. Ghorpade, S. Mantri, and D. Ghorpade, "Neural Networks For Face Recognition Using SOM,” vol. 4333, pp. 65-67, 2010.

[7]. N. Qu, J. Chen, J. Zuo, and J. Liu, "PSO-SOM Neural Network Algorithm for Series Arc Fault 
Detection," Adv. Math. Phys., vol. 2020, 2020, doi: 10.1155/2020/6721909.

[8]. S. Kim and J. Lee, "Facial Shape Recognition Using Self Organized Feature Map ( SOFM )," vol. 8, no. 4, pp. 104-112, 2019.

[9]. O. E. Dragomir, F. Dragomir, and M. Radulescu, "Matlab application of Kohonen Self-Organizing Map to classify consumers' load profiles," Procedia Comput. Sci., vol. 31, pp. 474-479, 2014, doi: 10.1016/j.procs.2014.05.292.

[10]. R. Mohanty and M. V Raghunadh, "Skin Color Segmentation based Face Detection using Multi-Color Space," vol. 5, no. 5, pp. 470-475, 2016, doi: 10.1021/la900547b.

[11]. C. Q. Lai and S. S. Teoh, "An efficient method of HOG feature extraction using selective histogram bin and PCA Feature reduction,” Adv. Electr. Comput. Eng., vol. 16, no. 4, pp. 101-108, 2016, doi: 10.4316/AECE.2016.04016.

[12]. W. Zhou, S. Gao, L. Zhang, and X. Lou, "Histogram of Oriented Gradients Feature Extraction from Raw Bayer Pattern Images,” IEEE Trans. Circuits Syst. II Express Briefs, vol. 67, no. 5, pp. 946-950, 2020, doi: 10.1109/TCSII.2020.2980557.

[13]. L. Wang, "Research and Implementation of Machine Learning Classifier Based on KNN," IOP Conf. Ser. Mater. Sci. Eng., vol. 677, no. 5, 2019, doi: 10.1088/1757-899X/677/5/052038.

[14]. N. Ali, D. Neagu, and P. Trundle, "Evaluation of k-nearest neighbour classifier performance for heterogeneous data sets," SN Appl. Sci., vol. 1, no. 12, 2019, doi: 10.1007/s42452-019-1356-9.

[15]. L. Feng, H. Li, Y. Gao, and Y. Zhang, “A Color Image Segmentation Method Based on Region Salient Color and Fuzzy C-Means Algorithm,” Circuits, Syst. Signal Process., vol. 39, no. 2, pp. 586-610, 2020, doi: 10.1007/s00034-019-01126-w.

[16]. X. Zheng, Q. Lei, R. Yao, Y. Gong, and Q. Yin, "Image segmentation based on adaptive K-means algorithm,” Eurasip J. Image Video Process., vol. 2018, no. 1, 2018, doi: 10.1186/s13640-018-0309-3. 